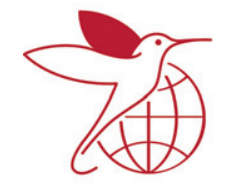

International

Diabetes

Federation

\title{
Serum visfatin and vaspin levels in normoglycemic first-degree relatives of Iranian patients with type 2 diabetes mellitus
}

\author{
Samad Akbarzadeh, Iraj Nabipour*, Seyed Mojtaba Jafari, Ali Movahed, Niloofar Motamed, \\ Majid Assadi, Najmeh Hajian
}

Department of Endocrine and Metabolic Diseases, The Persian Gulf Tropical Medicine Research Center, Bushehr University of Medical Sciences, Boostan 19 Alley, Imam Khomeini St, Bushehr 7514763448, Iran

\section{A R T I C L E I N F O}

\section{Article history:}

Received 5 December 2010

Received in revised form

11 September 2011

Accepted 3 October 2011

Published on line 22 October 2011

Keywords:

Adipocytokine

Beta cell

Insulin resistance

Insulin sensitivity

Diabetes mellitus

Obesity

\begin{abstract}
A B S T R A C T
Aim: To investigate circulating visfatin and vaspin levels in first-degree relatives of subjects with type 2 diabetes mellitus (FDRs) who frequently have higher value of HOMA-IR and beta cell dysfunction.

Methods: Serum visfatin and vaspin concentrations were measured in 179 Iranian subjects (90 normoglycemic FDRs and 89 age- and sex-matched healthy controls) using enzymelinked immunosorbent assay (ELISA) methods.

Result: Serum visfatin levels were significantly lower in the FDRs when compared to the controls $(1.71 \pm 0.93 \mathrm{ng} / \mathrm{ml}$ versus $2.69 \pm 2.02 \mathrm{ng} / \mathrm{ml}, p=0.0001)$. However, no significant difference was found in serum vaspin concentrations between the FDRs and the controls $(0.452 \pm 0.254 \mathrm{ng} / \mathrm{ml}$ versus $0.409 \pm 0.275 \mathrm{ng} / \mathrm{ml}, p>0.05)$. In multiple logistic regression analysis, the FDRs showed a significant association with lower visfatin levels after adjustments for age, sex, Body Mass Index, systolic and diastolic blood pressures, lipid profile, blood glucose levels and HOMA-IR [odds ratios $(\mathrm{OR})=1.71,95 \%$ confidence interval (1.30-2.25); $p<0.0001$.

Conclusion: The FDRs showed a significant association with lower visfatin levels. The observed lower circulating visfatin levels in FDRs may suggest a pathophysiological role for visfatin in beta cell dysfunction in this group.
\end{abstract}

(C) 2011 Elsevier Ireland Ltd. All rights reserved.

\section{Introduction}

Since the mid-1990s, several adipocytokines have been identified in relation to obesity and comorbidities, type 2 diabetes mellitus and its complications, metabolic syndrome, immunity, neuroendocrine, and cardiovascular functions [1-3]. The growing number of adipocyte-derived, cytokinelike hormones includes novel insulin resistance inducers and insulin-mimetic/insulin-sensitizer molecules such as adiponectin, leptin, visfatin, and vaspin.
Visfatin, which is identical to the "pre-B cell-colony enhancing factor" and "nicotinamide phosphoribosyltransferase," is secreted abundantly by the visceral fat of humans and mice and mimics the action of insulin [4]. Further, in vitro studies showed that glucose signaling for visfatin release in adipocytes involves the phosphatidylinositol 3-kinase (PI3kinase) and protein kinase $B$ pathway [5] and the regulation of glucose uptake by visfatin in human osteoblasts involves insulin receptor phosphorylation [6]. Although current data may suggest that insulin and visfatin act in concert to lower

\footnotetext{
* Corresponding author. Tel.: +98 7712541827; fax: +98 7712541828.

E-mail address: inabipour@gmail.com (I. Nabipour). 
blood glucose [7], human studies have shown inconsistent and conflicting results regarding associations between visfatin and insulin-mimetic effect, insulin resistance, beta cell function impairment, adiposity, subcutaneous versus visceral fat distribution, and diabetes $[1,3,8]$.

The plasma level of visfatin was associated with type 2 diabetes mellitus, independent of known risk factors, in a Chinese population [9]. Visfatin levels increased in Asian Indians with type 2 diabetes mellitus, but the association was lost after adjusting for obesity [10]. In another study, visfatin levels were reported to be higher in the diabetic group than the controls, but there was no significant difference in visfatin levels between the impaired glucose-tolerant group and the healthy controls [11]. Visfatin levels were correlated with HbA1c in patients with type 2 diabetes and lowered by intensive glycemic control [12]. Higher serum levels of visfatin in women with gestational diabetes were also reported $[13,14]$. Plasma visfatin concentration was negatively associated with vascular endothelial function in type 2 diabetes mellitus [15]. Visfatin was shown to be synthesized in cultured mesangial cells and to stimulate glucose uptake in glomerular mesangial cells. Thus, it was hypothesized that visfatin may contribute to acceleration of diabetic nephropathy through the aggravation of metabolic alterations [16].

Vaspin (visceral adipose tissue-derived serpin), a member of the serine protease inhibitor family, is a novel adipocytokine with insulin-sensitizing effects [17]. The circulating level and tissue expression of vaspin increased at the peak of obesity and insulin resistance in Otsuka Long-Evans Tokushima fatty (OLETF) rats, an animal model of abdominal obesity and type 2 diabetes mellitus [18]. It has been suggested that the compensatory increase in vaspin levels during insulin resistance may ameliorate the action of unknown up-regulated proteases, which impair the insulin action [18]. However, circulating vaspin levels in relation to insulin sensitivity/ insulin resistance status and glucose metabolism are conflicting and remain to be elucidated.

First-degree relatives of subjects with type 2 diabetes mellitus (FDRs) frequently have both insulin resistance [19] and beta cell dysfunction [20] and bear a $40 \%$ lifetime risk for developing type 2 diabetes mellitus [21]. With such a background of the pathophysiology of glucose metabolism in FDRs, investigating the circulating levels of novel adipocytokines, such as visfatin and vaspin, in this group is worthwhile. The present study, for the first time, sought to explore serum visfatin and vaspin concentrations in FDRs.

\section{Methods}

\subsection{Subjects and physical measurements}

A total of 90 glucose-tolerant subjects (41 men, 49 women, mean age $40.55 \pm 9.36$ years) of first-degree relatives of Iranian patients with type 2 diabetes mellitus (FDRs) who consecutively visited the Endocrine Clinic of Fatemeh-Zahra University Hospital were enrolled in this study. We selected an age- and sex-matched glucose-tolerant control group (49 men, 40 women, mean age $41.375 \pm 9.47$ years) from the Persian Gulf Healthy Heart Study participants [22] or subjects with no
Table 1 - The general characteristics, including blood pressure and anthropometric measurements, and the biochemical parameters of first-degree relatives of subjects with type 2 diabetes mellitus (FDRs) and healthy controls.

\begin{tabular}{|c|c|c|}
\hline & FDRs $(n=90)$ & Control $(n=89)$ \\
\hline Female/male ratio & $49 / 41$ & $40 / 49$ \\
\hline Age (years) & $40.55 \pm 9.63$ & $41.37 \pm 9.47$ \\
\hline Systolic BP (mmHg) & $115.0 \pm 13.8$ & $115.82 \pm 12.84$ \\
\hline Diastolic BP (mmHg) & $74.72 \pm 9.95$ & $74.45 \pm 10.47$ \\
\hline Height (cm) & $165.88 \pm 9.31$ & $166.89 \pm 8.87$ \\
\hline Weight (kg) & $75.92 \pm 13.43$ & $74.32 \pm 14.75$ \\
\hline Waist circumference $(\mathrm{cm})$ & $94.41 \pm 10.19$ & $93.10 \pm 12.63$ \\
\hline Hip circumference $(\mathrm{cm})$ & $104.76 \pm 9.975$ & $102.93 \pm 12.42$ \\
\hline WHR & $0.90 \pm 0.14$ & $0.91 \pm 0.17$ \\
\hline BMI $\left(\mathrm{kg} / \mathrm{m}^{2}\right)$ & $27.57 \pm 4.25$ & $26.52 \pm 4.24$ \\
\hline Fasting glucose (mg/dl) & $80.5 \pm 8.56$ & $79.77 \pm 10.46$ \\
\hline 2 h-OGTT (mg/dl) & $92.17 \pm 15.68$ & $92.03 \pm 16.65$ \\
\hline Total cholesterol (mg/dl) & $198.54 \pm 36.85$ & $196.92 \pm 37.24$ \\
\hline Triglyceride (mg/dl) & $168.51 \pm 82.3$ & $155.71 \pm 75.0$ \\
\hline HDL-cholesterol (mg/dl) & $43 \pm 10.38$ & $45.44 \pm 11.65$ \\
\hline LDL-cholesterol (mg/dl) & $121.01 \pm 31.55$ & $120.04 \pm 32.44$ \\
\hline Insulin $(\mu \mathrm{IU} / \mathrm{ml})$ & $8.75(5.25-16.68)$ & $6.56(5.03-10.18)^{*}$ \\
\hline HOMA-IR & $1.82(1.05-3.06)$ & $1.29(0.98-1.93)^{\circ}$ \\
\hline \multicolumn{3}{|c|}{$\begin{array}{l}\text { BMI, Body Mass Index; WHR, waist to hip ratio; BP, Blood Pressure; } \\
2 \text { h-OGTT, second-hour oral glucose tolerance test; HOMA-IR, } \\
\text { homeostasis model of assessment index. } \\
\text { Data are means (SD), except for insulin and HOMA-IR [medians } \\
\text { (interquartile ranges)]. } \\
\text { p values }<0.05 \text { (the FDRs in comparison to the controls). }\end{array}$} \\
\hline
\end{tabular}

apparent disease history, who underwent a routine health checkup at General Clinic of Fatemeh-Zahra University Hospital. They had no family history of diabetes mellitus, type 2 diabetes or endocrine disorders, cardiovascular diseases, or hepatic or renal dysfunction and were not taking antirheumatic drugs and medications for metabolic diseases. The participants in the control group had anthropometric measurements comparable to those of the FDRs (Table 1). All subjects were asked to fast and to present to the Persian Gulf Health Research Center between 7:30 and 9:30 a.m., after an overnight fast. They underwent a 75-g oral glucose tolerance test (OGTT). The definition of glucose tolerant was a subject who had a fasting plasma glucose level lower than $110 \mathrm{mg} / \mathrm{dl}$ and a second-hour blood sugar level after oral glucose load (2 h-OGTT) lower than $140 \mathrm{mg} / \mathrm{dl}$ [23].

Blood pressure was assessed twice at the right arm after a 15-min rest in the sitting position, using a standard mercury sphygmomanometer. Height and weight were measured using a stadiometer. Heavy outer garments and shoes were removed before height and weight were measured. Body Mass Index (BMI) was calculated. Waist circumference was defined at the midway level between the costal margins and the iliac crests. Hip circumference was measured at the level of the greater trochanters.

The study was approved by the medical-ethical committee of Bushehr University of Medical Sciences.

\subsection{Laboratory measurements}

A fasting blood sample was taken, all samples were promptly centrifuged, and sera were separated and kept frozen at $-70^{\circ} \mathrm{C}$ 
until used. Analyses for biochemical parameters (blood sugars, triglyceride, and cholesterol levels) were carried out at the Persian Gulf Health Research Center on the day of blood collection using a Selectra 2 autoanalyzer (Vital Scientific, Spankeren, the Netherlands). Glucose was assayed with the enzymatic (glucose oxidase) colorimetric method using a commercial kit (Pars Azmun Inc., Tehran, Iran). Serum total cholesterol and HDL (high-density lipoprotein) cholesterol were measured using cholesterol oxidase phenol aminoantipyrine and triglycerides using the glycerol-3 phosphate oxidase phenol aminoantipyrine enzymatic method. Serum LDL (low-density lipoprotein) cholesterol was calculated using the Friedwald formula; LDL cholesterol was not calculated when the triglycerides concentration was $>400 \mathrm{mg} / \mathrm{dl}$. Insulin was measured using a commercially available enzyme-linked immunosorbent assay kit (Insulin ELISA, DRG diagnostics, Marburg, Germany). The assay sensitivity was $1.76 \mu \mathrm{IU} / \mathrm{ml}$; the intra- and interassay coefficients of variance were $1.79-2.6 \%$ and $2.88-5.99 \%$, respectively. Insulin resistance was assessed by calculating the homeostasis model of assessment index (HOMA-IR) using the equation: fasting insulin ( $\mu \mathrm{IU} /$ $\mathrm{ml}) \times$ fasting glucose $(\mathrm{mg} / \mathrm{dl}) / 405$.

To detect visfatin and vaspin in the serum samples, commercially (Cat. No. V0523EK and Cat. No. V0712EK, respectively) available enzyme-linked immunosorbent assay kits (AdipoGen, Seoul, Korea) were used according to the manufacturer's instructions. The assay sensitivity for visfatin was $0.10 \mathrm{ng} / \mathrm{ml}$; the intra- and interassay coefficients of variance were $3.8-5.5 \%$ and $6.4-9.5 \%$, respectively. The assay sensitivity for vaspin was $0.012 \mathrm{ng} / \mathrm{ml}$; the intra- and interassay coefficients of variance were $1.3-3.8 \%$ and $3.3-9.1 \%$, respectively.

\subsection{Statistical analysis}

Normal distribution of the data was controlled with the Kolmogorov-Smirnov test. Probability values $<5 \%$ were considered statistically significant. The significance of the difference in the results between the two groups was determined with chi-square analysis using $2 \times 2$ contingency tables. A two-tailed t-test was used to compare the values across groups in the presence of a normal distribution. Significant differences were assessed with the Mann-Whitney $U$ test in the absence of a normal distribution. Multiple linear regression models were used to assess the association between adipocytokines (visfatin and vaspin) levels (independent variables) and anthropometric, blood pressure measurements, and biochemical variables (each factor was considered a separate dependent variable in a series of models). Because the distributions of serum insulin levels and HOMA-IR were skewed, logarithmically transformed values were used for statistical analysis. Insulin and HOMA-IR showed normal distributions after the logarithmic transformations. Binary logistic regression analysis was used to ascertain the associations between the visfatin levels and the presence of FDRs. Sex, age, BMI, systolic and diastolic blood pressures, glucose levels, HOMA-IR and lipid status parameters were considered covariates, and the presence of FDRs the dependent variable. Standard or simultaneous (Enter Method) type of regression analysis was used. All statistical analyses were performed using the PASW Statistics GradPack 18 (SPSS Inc., Chicago, IL).

\section{Results}

The general characteristics, including blood pressure and anthropometric measurements, and the biochemical parameters of the study groups are shown in Table 1. There was no significant difference between the two groups regarding blood pressure measurements, height, weight, waist and hip circumferences, waist to hip ratio, and BMI. There was also no significant difference in the fasting blood glucose, $2 \mathrm{~h}$-OGTT, and lipid profiles between the two groups. However, the FDRs had higher HOMA-IR and serum insulin levels than the control group $(p<0.05$, Table 1$)$.

Serum visfatin levels were significantly lower in the FDRs when compared to the controls $(1.71 \pm 0.93 \mathrm{ng} / \mathrm{ml}$ versus $2.69 \pm 2.02 \mathrm{ng} / \mathrm{ml}, p=0.0001)$. However, no significant difference was found in the serum vaspin concentrations between the FDRs and the controls $(0.452 \pm 0.254 \mathrm{ng} / \mathrm{ml}$ versus $0.409 \pm 0.275 \mathrm{ng} / \mathrm{ml}, p=0.305$; Fig. 1). No gender difference was found for the circulating visfatin and vaspin levels $(p>0.05)$.

There was no significant difference between the FDRs and control groups regarding the prevalence of consumption of antihypertensive agents (7.8\% versus $4.5 \%$ ), hypocholesterolemic drugs (6.7\% versus $5.6 \%$ ) and aspirin (5.6\% versus $7.9 \%$ ). A total of 10 women in the FDRs group and 13 women in the control group were on anticontraceptive pills.

Age- and sex-adjusted correlations for serum visfatin and vaspin in relation to anthropometric, biochemical, and blood pressure measures are shown in Table 2. Age- and sexadjusted serum visfatin concentration levels were significantly correlated with BMI in the FDRs $(\beta=0.22, p=0.04)$. However, there were no significant correlations between visfatin concentrations and HOMA-IR, the fasting blood glucose, $2 \mathrm{~h}$-OGTT, triglyceride, total cholesterol, HDL-cholesterol, LDL-cholesterol, insulin and vaspin levels in the FDRs. However, age- and sex-adjusted serum visfatin concentration levels were significantly correlated with total cholesterol

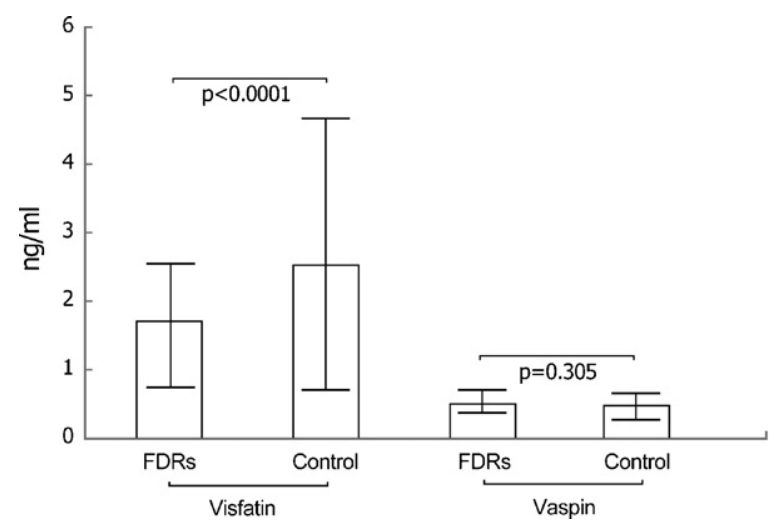

Fig. 1 - Serum visfatin and vaspin concentrations in firstdegree relatives of subjects with type 2 diabetes mellitus (FDRs, $n=90$ ) and healthy controls $(n=89)$. Data are means \pm standard deviation. 
Table 2 - Age- and sex-adjusted correlations for serum visfatin and vaspin in relation to anthropometric, biochemical, and blood pressure measures in first-degree relatives of subjects with type 2 diabetes mellitus (FDRs) and healthy controls.

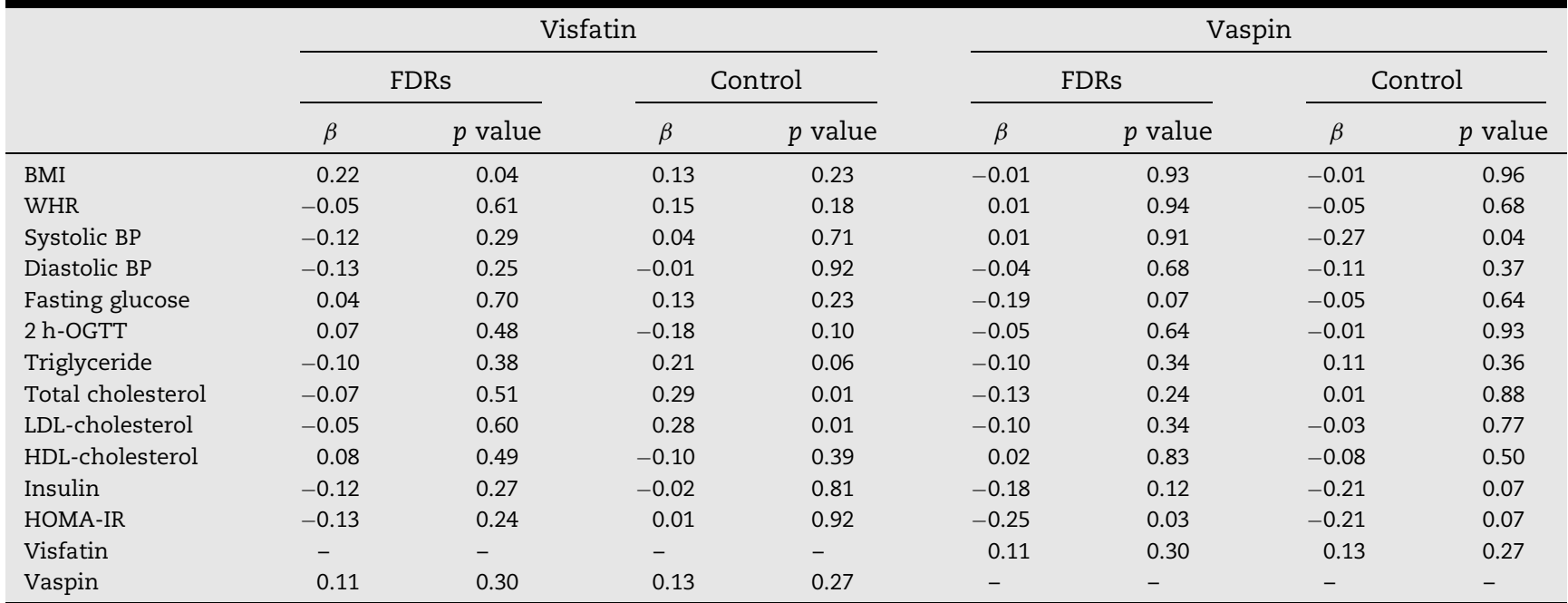

BMI, Body Mass Index; WHR, waist to hip ratio; BP, blood pressure; 2 h-OGTT, second-hour oral glucose tolerance test; HOMA-IR, homeostasis model of assessment index.

$(\beta=0.29, p=0.01)$ and LDL-cholesterol $(\beta=0.28, p=0.01)$ in the control group.

Age- and sex-adjusted vaspin was also not correlated with anthropometric, biochemical, or blood pressure measurements. However, age- and sex-adjusted serum vaspin concentration levels were significantly correlated with HOMA-IR in the FDRs $(\beta=-0.25, p=0.03)$.

Table 3 shows unadjusted and adjusted odds ratios (95\% CI) between the presence of FDRs and circulating adipocytokines levels. In multiple logistic regression analysis, the FDRs showed a significant association with lower visfatin levels, even after adjustments were made for age, sex, BMI, and biochemical parameters [OR $=1.71$, CI $(1.30-2.25) ; p<0.0001$ ]. However, after successive adjustment for potential confounders, the FDRs did not show a significant association with vaspin levels (Table 3).

\section{Discussion}

In the current study, we found that FDRs had a significant association with lower visfatin levels, even after adjusting for age, sex, BMI, and biochemical parameters in multivariate analyses. However, no significant difference was found in serum vaspin concentrations between the FDRs and the controls.

Visfatin shows some insulin mimetic properties [24], and its molecular mechanism of actions is under vigorous challenge [4,24,25]. Recently, Brown et al. [26] suggested that visfatin can significantly regulate insulin secretion, insulin receptor phosphorylation, and intracellular signaling and expression of a number of beta cell function-associated genes in mouse beta cells.

However, there are huge discrepancies in reports of visfatin in relation to insulin sensitivity and insulin resistance $[1,8,13]$. Similar to our study, a lack of correlation between circulating visfatin and insulin/HOMA [27,28] was reported, but in contrast, a significant correlation between visfatin levels and insulin/HOMA was observed by other authors $[9,13]$. Pagano et al. [28] reported that visfatin did not play a role in the development of insulin resistance, in human obesity, either as assessed by homeostasis model assessment or during lipid infusion. The findings of the latter study are in accordance with the results of other studies that did not find an association of serum visfatin with insulin resistance $[10,27,29]$. Taken together, the current knowledge does not

Table 3 - Unadjusted and adjusted odds ratios [OR; 95\% confidence interval (CI)] between the presence of first-degree relatives of subjects with type 2 diabetes mellitus (FDRs) and circulating adipocytokines (visfatin and vaspin) levels.

\begin{tabular}{|c|c|c|c|c|c|c|}
\hline & \multicolumn{3}{|c|}{ Visfatin } & \multicolumn{3}{|c|}{ Vaspin } \\
\hline & OR & CI & $p$ value & OR & CI & $p$ value \\
\hline Unadjusted & 1.53 & $1.22-1.93$ & $<0.0001$ & 0.53 & $0.16-1.76$ & 0.304 \\
\hline Adjusted for age + sex & 1.56 & $1.23-1.98$ & $<0.0001$ & 0.51 & $0.15-1.73$ & 0.282 \\
\hline Adjusted for age + sex + BMI & 1.60 & $1.25-2.06$ & $<0.0001$ & 0.50 & $0.14-1.73$ & 0.280 \\
\hline Adjusted for age + sex + WHR & 1.55 & $1.22-1.96$ & $<0.0001$ & 0.51 & $0.15-1.75$ & 0.287 \\
\hline Full model & 1.71 & $1.30-2.25$ & $<0.0001$ & 0.25 & $0.06-1.06$ & 0.061 \\
\hline
\end{tabular}

BMI, Body Mass Index; WHR, waist to hip ratio.

Full model included age, sex, BMI, systolic and diastolic blood pressures, HOMA-IR, fasting glucose, second-hour blood glucose, insulin, triglyceride, HDL-cholesterol and LDL-cholesterol levels and FDRs (yes versus no). 
suggest that visfatin plays an unequivocal role in the development of insulin resistance.

Likewise, visfatin's relationship with type 2 diabetes mellitus and its link with pathophysiological pathways in pre-diabetic states and metabolic syndrome are unclear, and there is inconsistency in the correlations between circulating visfatin and diabetes mellitus. In some studies, higher circulating visfatin levels in patients with type 2 diabetes mellitus $[9,11]$ and gestational diabetes mellitus $[13,14]$ were observed while lower levels of visfatin in patients with type 1 diabetes mellitus [30] and gestational diabetes mellitus [31] were reported. No correlation between visfatin and diabetes [15] independent of obesity [10] was found in other studies. Chen et al. [9] suggested that the compensatory elevated visfatin, which has potential insulin mimetic properties, in patients with diabetes mellitus, may reflect an impairment of its action in target tissues, dysregulation in its biosynthesis, or a response to hyperglycemia/hyperinsulinemia or the altered state of adipocytokines.

According to the above studies, it appears that frank impairment conditions of glucose metabolism (e.g., the diabetes state) are not good models for assessing the pathophysiological link of visfatin with diabetes mellitus. In the current study, we evaluated the circulating levels of visfatin in glucose-tolerant FDRs.

Impaired glucose metabolism is common in FDRs [19]. They also have defects in beta cell function at a time when they are not hyperglycemic, and this reduction affects insulin and amylin responses to glucose stimulation [20]. Since both insulin sensitivity and beta cell dysfunction predict conversion to diabetes [32,33], FDRs are at increased risk of type 2 diabetes [21]. A recent large population study [33] showed that beta cell impairment exists in the offspring of type 2 diabetes patients, even in the absence of insulin resistance, suggesting that beta cell dysfunction is the outstanding determinant for the development of diabetes mellitus in this group.

Revollo et al. [34] provided several lines of evidence that visfatin (Nampt)-mediated systemic nicotinamide adenine dinucleotide (NAD) biosynthesis is critical for beta cell function. Visfatin/Nampt is an important rate-limiting moiety in the NAD biosynthetic pathway from nicotinamide [35]. Alteration in NAD levels could alter the enzymatic activities of NDA-dependent deacetylase Sirt1 and affect important metabolic pathways that are essential for regulating glucosestimulated insulin secretion in pancreatic beta cells [34].

Revollo et al. [34] showed that visfatin/Nampt+/- mice and islets had defects in nicotinamide mononucleotide (NMN)/ NAD biosynthesis and glucose-stimulated insulin secretion, and these defects were corrected by the administration of NMN, a product of the visfatin reaction.

As a major limitation, beta cell function was not assessed in the current study. However, based on the results of the above study [34], we can hypothesize that the observed lower circulating visfatin levels in our FDRs may lead to derangement in visfatin-mediated systemic NAD biosynthesis and early defects in glucose-stimulated insulin secretion in their pancreatic beta cells. Undoubtedly, assessment of beta cell function and measurement of plasma NMN levels in FDRs would be helpful.
It should be noted that the linking mechanism of low visfatin levels and early beta cell dysfunction in FDRs appears not to be operative in the advanced phases of beta cell deterioration, similar to type 2 diabetes mellitus. In fact, increasing circulating visfatin levels was demonstrated with progressive beta cell deterioration [36]. To identify the pathophysiological stimuli that cause increased production of visfatin in type 2 diabetes, as reported by some authors, further investigations are needed.

We found a significant correlation between visfatin levels and BMI in the FDRs. Circulating visfatin concentration in relation to obesity is controversial $[27,28,37,38]$. In a recent study [39], visfatin/PBEF/Nampt mRNA levels were not correlated with measures of obesity, and the researchers suggested that visfatin is not predominantly secreted from visceral fat.

We found that age- and sex-adjusted serum visfatin concentration levels were significantly correlated with total cholesterol and LDL-cholesterol in the control group but this positive correlation was lost in the FDRs group. It was suggested that visfatin-induced free fatty acid flux to liver, drives very low density lipoprotein production, leading to elevated plasma triglyceride and total cholesterol [39]. However, there are controversies in reports of visfatin in relation to lipid metabolism. Positive correlations between visfatin concentrations and total cholesterol [39], HDLcholesterol [40,41] and triglyceride levels [39] were observed by other authors. In contrast, negative correlations between visfatin concentrations and LDL-cholesterol [40], HDLcholesterol and triglyceride [41] levels were reported. Zahorska-Markiewicz did not observe a correlation between visfatin concentrations and lipids in both the obese and lean groups [37]. The lack of correlation between visfatin levels and parameters of lipid metabolism in the FDRs group warrants further study.

In our study, there was no difference in circulating vaspin between the FDRs and the controls. Human vaspin expression was associated with obesity, insulin resistance, glucose metabolism in human [42] and insulin sensitivity in rats [43]. Vaspin levels were reported to be significantly elevated in nonobese and obese T2DM patients compared with controls [44]. In another study, there was no significant difference in circulating vaspin between normal glucose-tolerant subjects and patients with type 2 diabetes mellitus [45].

Although the association of vaspin levels with insulin resistance, obesity, and glycemic status is still controversial in humans [46], it seems that the production of vaspin from adipose tissue is a compensatory mechanism to ameliorate severe insulin resistance in obesity and type 2 diabetes $[42,45]$.

We did not measure insulin sensitivity with the goldstandard isoglycaemic-hyperinsulinemic clamp test in our subjects, which is a limitation; however, the FDRs did not show a significant association with vaspin levels after adjustments for HOMA-IR in multivariate analysis (Table 3). Since good correlations between HOMA-IR and insulin resistance assessed by some "gold standard" methods have been reported [47], we postulate that the level of insulin resistance in FDRs was not at a threshold to stimulate vaspin secretion, as a compensatory mechanism. 
We acknowledge several study limitations. As our data are cross-sectional, limited inferences can be made regarding temporality and causation. Another limitation of our study includes the lack of assessment of beta cell function. Since we assessed the investigated adipocytokines with single measurements, the changes in these adipocytokines over time could not be reflected in the current study. To clarify circulating adipocytokines in relation to worsening of glucose tolerance over time in FDRs, longitudinal studies are warranted. The measurement of additional adipocytokines and inflammatory markers and cytokines merits consideration to elucidate the complex system that regulates beta cell function and insulin sensitivity in pre-diabetes states.

In conclusion, there was no significant difference in circulating vaspin levels between the FDRs and the controls. Therefore, circulating vaspin might not have a determining value as a biological marker in FDRs. In contrast, the observed lower circulating visfatin levels in FDRs may suggest a pathophysiological role for visfatin in beta cell dysfunction in this group.

Because visfatin-mediated systemic NAD biosynthesis is vital for beta cell function [34], further studies are needed to elucidate the precise mechanisms behind underproduction of visfatin in FDRs to prevent further pathological consequences including development of type 2 diabetes mellitus in this group.

\section{Conflict of interest}

There are no conflicts of interest.

\section{Acknowledgments}

This study was supported in part by a grant from Bushehr Province Technology and Research Committee and Research Deputy of Bushehr University of Medical Sciences.

\section{R E F E R E N C E S}

[1] Lago F, Gómez R, Gómez-Reino JJ, Dieguez C, Gualillo O. Adipokines as novel modulators of lipid metabolism. Trends Biochem Sci 2009;34:500-10.

[2] Kralisch S, Bluher M, Paschke R, Stumvoll M, Fasshauer M. Adipokines and adipocyte targets in the future management of obesity and the metabolic syndrome. Mini Rev Med Chem 2007;7:39-45.

[3] Gulcelik NE, Usman A, Gürlek A. Role of adipocytokines in predicting the development of diabetes and its late complications. Endocrine 2009;36:397-403.

[4] Fukuhara A, Matsuda M, Nishizawa M, Segawa K, Tanaka M, Kishimoto K, et al. Visfatin: a protein secreted by visceral fat that mimics the effects of insulin. Science 2005;307:426-30.

[5] Haider DG, Schaller G, Kapiotis S, Maier C, Luger A, Wolzt M. The release of the adipocytokine visfatin is regulated by glucose and insulin. Diabetologia 2006;49:1909-14.

[6] Xie H, Tang SY, Luo XH, Huang J, Cui RR, Yuan LQ, et al. Insulin-like effects of visfatin on human osteoblasts. Calcif Tissue Int 2007;80:201-10.
[7] Katwa LC, Seidel ER. Visfatin in pregnancy: proposed mechanism of peptide delivery. Amino Acids 2009;37:555-8.

[8] Saddi-Rosa P, Oliveira CS, Giuffrida FM, Reis AF. Visfatin, glucose metabolism and vascular disease: a review of evidence. Diabetol Metab Syndr 2010;2:21.

[9] Chen MP, Chung FM, Chang DM, Tsai JC, Huang HF, Shin SJ, et al. Elevated plasma level of visfatin/pre-B cell colonyenhancing factor in patients with type 2 diabetes mellitus. J Clin Endocrinol Metab 2006;91:295-9.

[10] Sandeep S, Velmurugan K, Deepa R, Mohan V. Serum visfatin in relation to visceral fat, obesity, and type 2 diabetes mellitus in Asian Indians. Metabolism 2007;56:565-70.

[11] Dogru T, Sonmez A, Tasci I, Bozoglu E, Yilmaz MI, Genc H, et al. Plasma visfatin levels in patients with newly diagnosed and untreated type 2 diabetes mellitus and impaired glucose tolerance. Diabetes Res Clin Pract 2007;76:24-9.

[12] Zhu J, Schott M, Liu R, Liu C, Shen B, Wang Q et al. Intensive glycemic control lowers plasma visfatin levels in patients with type 2 diabetes. Horm Metab Res 2008;40: 801-5.

[13] Lewandowski KC, Stojanovic N, Press M, Tuck SM, Szosland K, Bienkiewicz M, et al. Elevated serum levels of visfatin in gestational diabetes: a comparative study across various degrees of glucose tolerance. Diabetologia 2007;50:1033-7.

[14] Krzyzanowska K, Krugluger W, Mittermayer F, Rahman R, Haider D, Shnawa N, et al. Increased visfatin concentrations in women with gestational diabetes mellitus. Clin Sci (Lond) 2006;110:605-9.

[15] Takebayashi K, Suetsugu M, Wakabayashi S, Aso Y, Inukai $\mathrm{T}$. Association between plasma visfatin and vascular endothelial function in patients with type 2 diabetes mellitus. Metabolism 2007;56:451-8.

[16] Song HK, Lee MH, Kim BK, Park YG, Ko GJ, Kang YS. Visfatin: a new player in mesangial cell physiology and diabetic nephropathy. Am J Physiol Renal Physiol 2008;295:F1485-94.

[17] Wada J. Vaspin: a novel serpin with insulin-sensitizing effects. Expert Opin Investig Drugs 2008;17:327-33.

[18] Hida K, Wada J, Eguchi J, Zhang H, Baba M, Seida A, et al. Visceral adipose tissue-derived serine protease inhibitor: a unique insulin-sensitizing adipocytokine in obesity. Proc Natl Acad Sci U S A 2005;102:10610-5.

[19] Eriksson J, Franssila-Kallunki A, Ekstrand A, Saloranta C, Widen, Schalin C, et al. Early metabolic defects in persons at increased risk for non-insulin-dependent diabetes mellitus. N Engl J Med 1989;321:337-43.

[20] Knowles NG, Landchild MA, Fujimoto WY, Kahn SESO. Insulin and amylin release are both diminished in firstdegree relatives of subjects with type 2 diabetes. Diabetes Care 2002;25:292-7.

[21] Perseghin G, Ghosh S, Gerow K, Shulman GI. Metabolic defects in lean nondiabetic offspring of NIDDM parents: a cross-sectional study. Diabetes 1997;46:1001-9.

[22] Nabipour I, Amiri M, Imami SR, Jahfari SM, Shafeiae E, Nosrati A, et al. The metabolic syndrome and nonfatal ischemic heart disease; a population-based study. Int J Cardiol 2007;118:48-53.

[23] Carnevale Schianca GP, Rossi A, Sainaghi PP, Maduli E, Bartoli $\mathrm{E}$. The significance of impaired fasting glucose versus impaired glucose tolerance: importance of insulin secretion and resistance. Diabetes Care 2003;26: 1333-7.

[24] Stephens JM, Vidal-Puig AJ. An update on visfatin/pre-B cell colony-enhancing factor, an ubiquitously expressed, illusive cytokine that is regulated in obesity. Curr Opin Lipidol 2006;17:128-31. 
[25] Fukuhara A, Matsuda M, Nishizawa M, Segawa K, Tanaka M, Kishimoto K, et al. Retraction. Science 2007;318:565.

[26] Brown JE, Onyango DJ, Ramanjaneya M, Conner AC, Patel ST, Dunmore SJ, et al. Visfatin regulates insulin secretion, insulin receptor signalling and mRNA expression of diabetes-related genes in mouse pancreatic beta-cells. J Mol Endocrinol 2010;44:171-8.

[27] Berndt J, Klöting N, Kralisch S, Kovacs P, Fasshauer M, Schön MR, et al. Plasma visfatin concentrations and fat depot-specific mRNA expression in humans. Diabetes 2005;54:2911-6.

[28] Pagano C, Pilon C, Olivieri M, Mason P, Fabris R, Serra R, et al. Reduced plasma visfatin/pre-B cell colony-enhancing factor in obesity is not related to insulin resistance in humans. J Clin Endocrinol Metab 2006;91:3165-70.

[29] Hammarstedt A, Pihlajamäki J, Rotter Sopasakis V, Gogg S, Jansson PA, Laakso M, et al. Visfatin is an adipokine, but it is not regulated by thiazolidinediones. J Clin Endocrinol Metab 2006;91:1181-4.

[30] Toruner F, Altinova AE, Bukan N, Arslan E, Akbay E, Ersoy R, et al. Plasma visfatin concentrations in subjects with type 1 diabetes mellitus. Horm Res 2009;72:33-7.

[31] Chan TF, Chen YL, Lee CH, Chou FH, Wu LC, Jong SB, et al. Decreased plasma visfatin concentrations in women with gestational diabetes mellitus. J Soc Gynecol Investig 2006;13:364-7.

[32] Warram JH, Martin BC, Krolewski AS, Soeldner JS, Kahn CR. Slow glucose removal rate and hyperinsulinemia precede the development of type II diabetes in the offspring of diabetic parents. Ann Intern Med 1990;113:909-15.

[33] Stadler M, Pacini G, Petrie J, Luger A, Anderwald C, RISC Investigators. Beta cell (dys)function in non-diabetic offspring of diabetic patients. Diabetologia 2009;52:2435-44.

[34] Revollo JR, Körner A, Mills KF, Satoh A, Wang T, Garten A, et al. Nampt/PBEF/Visfatin regulates insulin secretion in beta cells as a systemic NAD biosynthetic enzyme. Cell Metab 2007;6:363-75.

[35] Revollo JR, Grimm AA, Imai S. The NAD biosynthesis pathway mediated by nicotinamide phosphoribosyltransferase regulates Sir2 activity in mammalian cells. J Biol Chem 2004;279(3):50754-63.

[36] López-Bermejo $\mathrm{A}$, Chico-Julià $\mathrm{B}$, Fernàndez-Balsells $\mathrm{M}$, Recasens M, Esteve E, Casamitjana R, et al. Serum visfatin increases with progressive beta-cell deterioration. Diabetes 2006;55:2871-5.
[37] Zahorska-Markiewicz B, Olszanecka-Glinianowicz M, Janowska J, Kocełak P, Semik-Grabarczyk E, Holecki M, et al. Serum concentration of visfatin in obese women. Metabolism 2007;56:1131-4.

[38] Haider DG, Schindler K, Schaller G, Prager G, Wolzt M, Ludvik B. Increased plasma visfatin concentrations in morbidly obese subjects are reduced after gastric banding. J Clin Endocrinol Metab 2006;91:1578-81.

[39] Chang YC, Chang TJ, Lee WJ, Chuang LM. The relationship of visfatin/pre-B-cell colony-enhancing factor/ nicotinamide phosphoribosyltransferase in adipose tissue with inflammation, insulin resistance, and plasma lipids. Metabolism 2010;59:93-9.

[40] Chen CC, Li TC, Li CI, Liu CS, Lin WY, Wu MT, et al. The relationship between visfatin levels and anthropometric and metabolic parameters: association with cholesterol levels in women. Metabolism 2007;56:1216-20.

[41] Wang P, van Greevenbroek MM, Bouwman FG, Brouwers MC, van der Kallen CJ, Smit E, et al. The circulating PBEF/NAMPT/ visfatin level is associated with a beneficial blood lipid profile. Pflugers Arch 2007;454:971-6.

[42] Klöting N, Berndt J, Kralisch S, Kovacs P, Fasshauer M, Schön MR, et al. Vaspin gene expression in human adipose tissue: association with obesity and type 2 diabetes. Biochem Biophys Res Commun 2006;339:430-6.

[43] Wang YM, Wang WP, Wang LP, Lü QH, Zhou XH. Calorie control increased vaspin levels of serum and periepididymal adipose tissue in diet-induced obese rats in association with serum free fatty acid and tumor necrosis factor alpha. Chin Med J (Engl) 2010;123:936-41.

[44] El-Mesallamy HO, Kassem DH, El-Demerdash E, Amin AI. Vaspin and visfatin/Nampt are interesting interrelated adipokines playing a role in the pathogenesis of type 2 diabetes mellitus. Metabolism 2010. June 1 [Epub ahead of print].

[45] Youn BS, Klöting N, Kratzsch J, Lee N, Park JW, Song ES, et al. Serum vaspin concentrations in human obesity and type 2 diabetes. Diabetes 2008;57:372-7.

[46] Chang HM, Park HS, Park CY, Song YS, Jang YJ. Association between serum vaspin concentrations and visceral adipose tissue in Korean subjects. Metabolism 2010;59:1276-81.

[47] Song Y, Manson JE, Tinker L, Howard BV, Kuller LH, Nathan L, et al. Insulin sensitivity and insulin secretion determined by homeostasis model assessment and risk of diabetes in a multiethnic cohort of women: the Women's Health Initiative Observational Study. Diabetes Care 2007; 30:1747-52. 